\title{
Estudio hermenéutico del entrenamiento Físico militar a nivel mundial
}

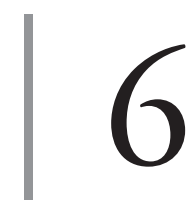

https://doi.org/10.21830/9789585284814.06

\author{
Ana Isabel García Muñoz', Paula Janyn Melo Buitrago', Laura Elizabeth \\ Castro Jiménez ${ }^{3}$, Daniel Fernando Aparicio Gómez ${ }^{4}$
}

\section{Resumen}

Objetivo: develar los principales logros y limitaciones que se evidencian en publicaciones científicas sobre el entrenamiento físico militar en los últimos 10 años, mediante un estudio hermenéutico. Metodología: se planteó un estudio con enfoque cualitativo desde una postura crítico-hermenéutica, siguiendo el modelo de revisión ampliada de la literatura; para la selección de los artículos se tuvo en cuenta criterios de inclusión y exclusión con una ventana de observación de los últimos 10 años, aplicando una ecuación de búsqueda utilizando términos MeSH en las bases de datos Pubmed, Biblioteca Virtual de salud (Lilacs, Scielo, Cochrane) y Scopus. Luego se realizó la sistematización de los artículos en una matriz de Excel definiendo en cada artículo de manera relevante si se relacionaba con la categoría de análisis, que corresponde al entrenamiento físico militar. Resultados: la búsqueda inicial dio como resultado 570 artículos, de los cuales 18 de Pubmed, 7 de la Biblioteca virtual de salud y 5 de Scopus fueron seleccionados para el análisis. Como categorías emergentes se identificaron: características del entrenamiento militar, objetivos del entrenamiento militar, métodos del entrenamiento y lesiones, evaluación del efecto del entrenamiento y adelantos tecnológicos. Conclusiones: los logros observados con el

1 Terapeuta respiratoria, especialista en Rehabilitación cardiopulmonar, especialista en docencia universitaria. Magister en desarrollo educativo y social. Grupo de Investigación RENFIMIL, Escuela Militar de cadetes General José María Córdova. Contacto: ana.garcia@esmic.edu.co. Orcid: https://orcid. org/0000-0003-4455-4534

2 Licenciada en Educación Física, Especialista en Docencia Universitaria, Magister Educación con énfasis en Pedagogía del Entrenamiento Deportivo. Coordinadora de Investigaciones Facultad de Educación Física militar. Grupo de investigación RENFIMIL - Escuela Militar de Cadetes General José María Córdova. Contacto: paula.melo@esmic.edu.co. Orcid: https://orcid.org/0000-0002-3492-7985

3 Fisioterapeuta. Magister en Salud Pública. Doctorando en Humanidades, Humanismo y Persona. Docente Investigadora Grupo Cuerpo, Sujeto y Educación. Universidad Santo Tomás. Contacto: laura.castro@usantotomas.edu.co. Orcid: https://orcid.org/0000-0001-5166-8084

4 Médico, Especialista en medicina del deporte. Magister en Ciencias y Tecnologías del Deporte y la Actividad Física. Grupo de investigación RENFIMIL, Escuela Militar de Cadetes General José María Córdova. Contacto: daniel.aparicio@esmic.edu.co. Orcid: https://orcid.org/0000-0001-5574-7580 
entrenamiento militar dependen del tipo, frecuencia, intensidad y duración del mismo, así como de las características del contexto donde se realicen, lo que, en muchos casos, es un aspecto que se convierte en una limitante, como lo es también la indumentaria que en algunas ocasiones se debe usar y los cortos periodos de sueño y descanso.

Palabras clave: educación; entrenamiento físico; personal militar; revisión.

\section{Introducción}

El entrenamiento físico militar busca la afinación de las cualidades morfofuncionales de sus hombres y mujeres en pro de optimizar su rendimiento terrestre, aéreo, acuático y aeroespacial, en los diferentes contextos donde son ejecutados, ya sea en desierto, alta montaña, páramo o montañoso (Fedak, 2014). En tal sentido, pretende contribuir, en lo que a aptitud física se refiere, a generar

una fuerza multimisión interoperable, capaz de garantizar a la Nación, cobertura estratégica, control territorial y protección de la población civil, de los activos estratégicos, de las zonas económicas y productivas, del medio ambiente y de los recursos naturales, además de garantizar el despliegue internacional. (Rojas, 2017, p. 100).

Todo lo anterior, sin descuidar los objetivos deportivos de sus atletas. En este escenario, la preparación física para el combate toma un papel protagónico, tal y como lo sugiere Podestá (2016), por cuanto de ella depende el éxito o fracaso de las misiones y los años de vida militar útil. Frente a esta necesidad, han surgido varios estudios que recomiendan diversos esquemas de entrenamiento que combinan, entre otros, el entrenamiento de resistencia con el de fuerza, a diferentes intensidades, en cursos básicos avanzados de combate, siendo importante realizar un análisis interpretativo de sus indicaciones y efectos, con el fin de reconocer cuáles de ellos han evidenciado optimización del desempeño con menor número de lesiones asociadas.

Es así como el estudio hermenéutico cobra un papel preponderante, ya que el paradigma cualitativo y documental que lo caracteriza permite abordar textos y poner en diálogo las perspectivas de diferentes autores en torno a un tema para, a partir de ellas, generar comprensiones más amplias. En este caso y bajo esta perspectiva, se estudió el entrenamiento físico miliar, buscando develar sus principales logros y limitaciones. 


\section{Metodología}

Se planteó un estudio con enfoque cualitativo desde una postura critico-hermenéutica en el cual los investigadores realizaron una interpretación de los discursos existentes y generaron interpretaciones nuevas a la luz de lo referenciado por los autores, siguiendo el modelo de revisión ampliada de la literatura. En los criterios de inclusión se determinó el análisis de artículos publicados en revistas indexadas, originales, en la ventana de tiempo establecida - 10 años - y que tuvieran la información requerida sobre entrenamiento militar. Se excluyeron los artículos de consenso, opinión de expertos y aquellos que no permitieran evidenciar los logros y limitaciones del entrenamiento. Para ello se planteó una ecuación de búsqueda utilizando palabras $\mathrm{MeSH}^{5}$ en las siguientes bases de datos: Pubmed, Biblioteca Virtual de salud (Lilacs, Scielo, Cochrane) y Scopus.

Los resultados de la búsqueda inicial se almacenaron en una matriz en Excel, en la cual se incluyó información relacionada con el título, el autor, metodología utilizada y si se relacionaba con la categoría de análisis definida para esta investigación: entrenamiento físico militar. Previamente, el equipo investigador se reunió y definió la categoría como: desarrollo intencionado de las capacidades psicofísicas de un individuo mediante una carga de entrenamiento, con una duración y frecuencia suficientes para provocar un efecto observable y medible, con el objetivo de responder a las demandas ocupacionales del contexto militar.

Se realizó un empalme entre los artículos que definen el entrenamiento físico militar y la definición concertada por el equipo investigador, se prosiguió entonces a hacer un análisis hermenéutico-crítico, el cual es "un ámbito para comprender críticamente al proceso de racionalidad vital, se trata entonces de proponer nuevos fundamentos teóricos" (Mardones, 1991) a partir del análisis e interpretación de la literatura.

5 ("physical education and training"[MeSH Terms] OR ("physical"[All Fields] AND "education"[All Fields] AND "training"[All Fields]) OR "physical education and training"[All Fields]) AND ("military personnel"[MeSH Terms] OR ("military"[All Fields] AND "personnel”[All Fields]) OR "military personnel”[All Fields]) AND (“loattrfull text”[sb] AND “2007/10/22”[PDat] : “2017/10/18”[PDat]) 
Con el análisis de los resultados emergieron las siguientes categorías, las cuales se tuvieron en cuenta en la presentación de resultados:

1. Características particulares del entrenamiento en militares.

2. Objetivos del entrenamiento militar.

3. Adelantos tecnológicos.

4. Métodos de entrenamiento y lesiones.

5. Evaluación del efecto del entrenamiento.

\section{Resultados y discusión}

La búsqueda inicial en las diferentes bases de datos dio como resultado 324 artículos en Pubmed, 123 artículos en Biblioteca Virtual de salud (Bvs) y en Scopus 123 artículos; para un total de 570 documentos revisados. Posteriormente, se procedió a la depuración de artículos, a partir de la definición de la categoría "entrenamiento físico militar", formulada por el grupo de investigadores. De esta manera, se identificaron 18 artículos de Pubmed, 7 artículos bvs y 5 en Scopus. Del análisis de estos materiales se presentan los resultados por categorías.

\section{Características particulares del entrenamiento en militares}

Los militares requieren desarrollar una variedad de cualidades físicas para satisfacer las demandas de la profesión militar, así mismo deben ser capaces de recuperarse de las demandas de la misión y del entrenamiento extenuante en corto tiempo (Szivak \& Kraemer, 2015; Gonçalves, Fonseca, Beltrame \& Correa, 2016), situación que lleva a un estrés crónico que reduce el rendimiento de la misión y aumenta el riesgo de lesiones. Si a esta situación se suma inapropiado entrenamiento, nutrición inadecuada y falta de sueño, el riesgo de padecer síndrome metabólico, resistencia a la insulina e hipertensión, será alto, afectando también la memoria y la función cognitiva (Lisman, O’Connor, Deuster, \& Knapik, 2013; Crowley et al., 2012). De hecho, se ha documentado que, aunque el ejercicio es una herramienta importante en la prevención del riesgo cardiovascular (Nogueira et al., 2016), en los atletas de élite y 
los militares puede llegar a elevar tal riesgo (Gonçalves, Fonseca, Beltrame \& Correa, 2016), incluso desde el inicio de la vida militar.

Dentro de los tipos de entrenamiento militar se ha utilizado predominantemente el entrenamiento de resistencia como principal modo de acondicionamiento físico aeróbico, con énfasis histórico puesto en la carrera de larga distancia (Gibala, Gagnon \& Nindl, 2015). El ejercicio de resistencia, en particular, confiere muchos beneficios para incluir una mayor fuerza y potencia, una mejor composición corporal y efectos protectores en tendones, ligamentos y huesos, favoreciendo con ello la resistencia a factores estresantes operacionales durante el combate. Así mismo, este tipo de entrenamiento induce cambios favorables en el press de banca y sentadilla en 1 repetición máxima, flexiones, abdominales y en el tiempo para completar una distancia de $30 \mathrm{~m}$.

Pese a los efectos del entrenamiento en resistencia, el entrenamiento por intervalos — caracterizado por episodios breves e intermitentes de ejercicio intenso, separados por periodos de ejercicio de menor intensidad o descanso para la recuperación - provoca adaptaciones cardiorrespiratorias y metabólicas más rápidas. De igual modo, se ha evidenciado que el entren amiento de dos semanas en resistencia, con carrera de 3000 metros o con intervalos de alta intensidad de $4 \times 4$ minutos o con entrenamiento continuo, mejora el rendimiento de los reclutas en la carrera (Riiser, Ripe \& Aadland, 2015). Así mismo, la distribución del entrenamiento semanal en varias sesion es cortas, en vez de menos sesiones más largas, mejora la ganancia de fuerza máxima sin comprometer la absorción máxima de oxígeno (Kilen et al., 2015). Es por ello que la combinación de ambos entrenamientos sería lo recomendado para labores terrestres, mientras que para personal de las Fuerzas Armadas moderadamente entrenado sin acceso a los equipos, el entrenamiento físico High Intensity Training (НIT) —con ejercicios de cuerda corporal que involucren de 4 a 7 series de 30 segundos, durante 4 semanas, 3 días por semana con 60 minutos - mantiene la forma física a pesar de la corta duración y el volumen reducido de actividad (Gist, Freese, Ryan \& Cureton, 2015).

Si bien los efectos positivos del HIT han sido ampliamente demostrados, no sucede lo mismo cuando se adicionan cargas, pues hacerlo se asocia con menor rendimiento y con mayor posibilidad de lesiones, cuando se realizan 
cambios de posición como pasar de prono a correr. Por ello, el entrenamiento simulado con carga ofrece muy poco o ningún beneficio en militares poco entrenados, mientras que los atletas anaerobios de élite, entrenados durante más o menos 3 semanas en hipergravedad en las actividades diarias, sí mejoran el rendimiento (O'Neal, Hornsby \& Kelleran, 2014). De otra parte, la organización temporal de la carga de entrenamiento no representa diferencias en cuanto al rendimiento militar, así lo evidenció un ensayo clínico en el cual se comparó en un grupo de soldados, con al menos 1 año en el Ejército, los efectos de 4 diferentes mesociclos de entrenamiento sobre el rendimiento, concluyendo que cualquiera de estos entrenamientos produce mejoras significativas para todas las variables dependientes.

En línea directa con el entrenamiento militar, el entrenamiento de la propiocepción se ha considerado vital para el adecuado funcionamiento de las extremidades inferiores. $\mathrm{Al}$ respecto, se demostró que 4 semanas de ejercicios propioceptivos durante 10 minutos diarios favorecen los movimientos repetidos en superficies inestables, con menor riego de lesiones y mejoría del equilibrio postural estático y dinámico después de la fatiga (Vantarakis et al., 2017).

En lo que respecta al entrenamiento de la fuerza relativa, se ha documentado que con entrenamiento tres veces por semana durante 5 semanas, acompañado del entrenamiento básico de preparación física militar, se mejora la carrera de $20 \mathrm{~m}$, sin que llegue a representar efectos estadísticamente distintos en el salto horizontal de pie, pull-ups y prueba de sentarse y alcanzar, mientras que el entrenamiento de la resistencia continua programada, acompañado del entrenamiento básico, sí mejora de manera estadísticamente significativa estas variables (Sporiš et al., 2014).

Por otra parte, el entrenamiento de agilidad se ha instituido recientemente en varias comunidades militares con la esperanza de mejorar el rendimiento en combate y la forma física general, demostrando mejoras significativas en el consumo submáximo de $\mathrm{O}_{2}$ en la prueba de agilidad de Illinois, en la de vigilancia visual y en la memoria continua (Lennemann et al., 2013).

\section{Objetivos del entrenamiento militar}

Dadas las circunstancias y que los principales objetivos del entrenamiento militar y naval son el desarrollo de la preparación, el rendimiento y la preven- 
ción de lesiones (Vantarakis et al., 2017), las organizaciones militares en todo el mundo hacen esfuerzos continuos para mejorar los programas de entrenamiento físico de sus Ejércitos, lo que hace necesario una adecuada interacción entre los métodos de entrenamiento militar básico (Gonçalves et al., 2016), así como un cambio en la asignación de los tiempos de descanso y recuperación por parte de los comandos, para prevenir lesiones. Por ello, el entrenamiento físico debe fomentar el desarrollo de capacidades de fuerza y potencia para un rendimiento óptimo de las tareas anaeróbicas, como el transporte de cargas pesadas, correr bajo carga y maniobrar en terrenos irregulares, los cuales deben ser adaptados a una perspectiva más moderna, tal como lo hacen los atletas anaerobios de fuerza de élite en los deportes actuales, en donde el entrenamiento individualizado es una realidad posible (Kraemer \& Szivak, 2012). Esto, debido a que los tipos de ejercicio, distancias y estándares varían para cada rama militar, aunque todos los miembros del servicio deban cumplir con un requisito mínimo semestral o anual (Lisman et al., 2013).

En lo que atańe a la necesidad de individualizar el entrenamiento, también es importante trabajar en este aspecto cuando se entrena a mujeres, pues por décadas se ha limitado su participación en algunas misiones al darse por cierto que la fuerza en sus miembros superiores es inferior a la de los hombres, cuando se ha evidenciado ya que algunas marines han podido completar las tareas más desafiantes con relación al fortalecimiento de la parte superior del cuerpo, lo que sugiere que todo depende de la especificidad en el entrenamiento (Jameson, Pappa, McGuire \& Kelly, 2015).

Un objetivo clave del entrenamiento militar consiste en lograr una capacidad cardiovascular y neuromuscular que permita una mejora significativa de la aptitud aeróbica, es así como luego de 8 semanas de entrenamiento básico seguidas por 8 semanas de entrenamiento militar especializado, en una cohorte de militares al inicio de su vida militar, se logró una mejoría en el consumo máximo de oxígeno $\left(\mathrm{VO}_{2 \text { pico }}\right)$ y en la fuerza isométrica bilateral máxima de la pierna y los músculos extensores del brazo, así como una disminución en el porcentaje de grasa corporal y la circunferencia de la cintura; por ello, se recomienda incluir un entrenamiento físico estructurado con mayor intensidad, aumento en volumen del mismo y una periodización óptima. 
De otra parte, es importante tener presente el estado de ánimo, a este respecto, en algunos países, como en el caso de Suiza, el uso de entrenamiento de circuito al aire libre como una adición al entrenamiento estándar —además de conducir a mejoras significativas en la aptitud física, fuerza muscular y control postural, sin incremento en las lesiones (Hofstetter, Mader \& Wyss, 2012) — contrarresta los efectos perjudiciales del estrés, aspecto sobre el cual los desarrollos tecnológicos han venido trabajando, lo que ha generado programas que, además de incluir el entrenamiento en cualidades físicas y cognitivas, producen cambios positivos en el estado de ánimo de ambos sexos (Lieberman et al., 2016).

\section{Adelantos tecnológicos}

El Eagle Tactical Athlete Program (ETAP) —Entrenamiento físico dirigido no comisionado-, desarrollado para la 101 División Aerotransportada del Ejército de EE. UU. (Asalto Aéreo) para contrarrestar las lesiones musculo-esqueléticas no intencionales, demostró una reducción significativa en la proporción de soldados con fracturas por estrés. De otra parte, los ambientes simulados para entrenamiento militar han ido cobrando un papel importante, siempre y cuando contemplen aspectos humanos, sociales y culturales del mundo real, así como las condiciones físicas del terreno, las características estructurales, los modelos físicos y los modelos de comportamiento, para interactuar de manera más efectiva y con respuestas intuitivas en situaciones sociales complejas. En tales ambientes simulados sería importante evaluar aspectos biomecánicos relacionados con el entrenamiento físico, en este punto también es importante resaltar la ventaja de la tecnología con sensores en la evaluación de pasos y distancia alcanzados, pues resulta más eficiente que el uso de instrumentos tradicionales que realizan una medición única (Wyss, Scheffler \& Mäder, 2012).

\section{Métodos de entrenamiento y lesiones}

La mayoría de los soldados obtendrán los mayores beneficios de los programas de entrenamiento físico y mental, combinados con buenos principios de descanso y nutrición (Friedl, 2015). Los métodos de entrenamiento 
no convencionales, como el entrenamiento de fuerza de las extremidades inferiores, entrenamiento CrossFit, entrenamiento con pesas rusas y entrenamiento de agilidad, son prometedores para mejorar la condición aeróbica y mejorar los puntajes de la condición física, especialmente entre los miembros que tienen dificultades para pasar una prueba de aptitud física. Sin embargo, desafortunadamente, la mayoría de las formas no tradicionales de entrenamiento físico no se admiten en la literatura científica, con excepción del entrenamiento de fuerza de las piernas y el entrenamiento de agilidad, por lo que son necesarios más estudios en esta área (O’Hara et al., 2012).

Si bien es cierto que el riesgo físico es inherente a las operaciones especiales, las lesiones no traumáticas resultantes del uso excesivo, la biomecánica deficiente y la selección arbitraria de ejercicios, pueden prevenirse con entrenamiento físico que reconozca la continuidad entre la rehabilitación y el entrenamiento de rendimiento, para garantizar que las adaptaciones fisiológicas no se realicen a expensas de la salud ortopédica o el dominio del movimiento, del tal manera que se optimice el éxito de la misión, la calidad de vida y la longevidad del militar. Como afirman Kechijian y su equipo de trabajo, a la luz de cuánto está en juego, el cuidado de la medicina deportiva en el Ejército, especialmente para las operaciones especiales, debe ser similar al que se practica en el atletismo profesional o en el universitario (Kechijian \& Rush, 2012; Larsson, Tegern \& Harms-Ringdahl, 2012).

Teniendo en cuenta que se han identificado patrones de movimiento anormales como importantes factores de riesgo prospectivo para la lesión de la extremidad inferior, incluida la lesión del ligamento cruzado anterior, es necesario que antes de programar un entrenamiento se evalúen los patrones de movimiento de "alto riesgo" que prevalecen en todos los niveles de atletas y, por tanto, en deportistas militares (Theiss et al., 2014). A su vez, resulta importante que a la hora de programar el entrenamiento físico se contemplen aquellos aspectos individuales y contextuales que podrían influir en el desempeño (Vierling, Whittington \& Cross, 2012).

\section{Evaluación del efecto del entrenamiento}

Dentro de las pruebas físicas empleadas para valorar el nivel de condición física, se emplean, entre otros, el salto de longitud, pull-ups, sentadillas y carrera 
de 1000 m (Tomczak, Bertrandt, Klos \& Klos, 2016). Las pruebas de aptitud física (resistencia aeróbica, el esfuerzo máximo en 1.5 a 3 millas o nadando, la resistencia muscular, como abdominales cronometrado, sentadillas y flexiones o pull-ups hasta el agotamiento) y las pruebas de aptitud para combate (test con taladro de carga de tanques de $120 \mathrm{~mm}$, carga de artillería redonda de $155 \mathrm{~mm}$, muro de obstáculos con una carga de combate ( $\approx 30 \mathrm{lb})$, pull-ups, peso muerto y limpieza y prens), son utilizadas por los servicios militares para confirmar que los miembros del servicio tienen niveles adecuados para cumplir el deber militar que se les ha asignado (Lisman et al., 2013). Escenario en el cual las mujeres han demostrado suficiencia para satisfacer las demandas de las ocupaciones de combate cerrado.

En algunas actividades, como los cursos de paracaidismo, se ha evidenciado una tasa de retiro alta dadas sus exigencias, por ello se han implementado una serie de pruebas, como la de optimización de entrenamiento (TOPtest), que utiliza dos eventos de ejercicio máximo para evaluar los cambios en los parámetros, antes y después, del entrenamiento (Vrijkotte et al., 2017). Con este test ha sido posible evidenciar una correlación directa entre los valores de frecuencia cardiaca, tensión y fatiga alcanzados en el entrenamiento y la deserción del curso.

Con lo descrito hasta aquí, resulta evidente la necesidad de introducir en la evaluación del entrenamiento militar actual la medición de la fuerza y resistencia de miembros inferiores, adoptando los test que ya se han venido empleando y que han arrojado notables beneficios, pues disminuyen las lesiones y el desgaste de los reclutas, al tiempo que reducen con éxito las tasas de abandono del servicio militar (Roos et al., 2015).

\section{Conclusiones}

Con referencia a la bibliografía consultada, se evidencia como logro del entrenamiento físico militar la mejora y optimización de las condiciones físicas del individuo en pro de la preparación para las operaciones militares y el combate, no siendo siempre evidente el mejoramiento de todas las aptitudes físicas del individuo, como, por ejemplo, en algunos entrenamientos en donde no se evidenciaron cambios estadísticamente significativos, sin querer 
decir con esto que no se mejoren posiblemente sus bases tácticas o técnicas (las cuales no son evaluadas en este estudio).

Se evidencia, también como logro, que el entrenamiento de resistencia de tipo continuo y el entrenamiento interválico tipo HIT y en circuito, sumados a los entrenamientos de coordinación y propiocepción, mejoran algunas cualidades de la aptitud física (fitness) del militar, además de lograr mejoras en composición corporal y disminuir el riesgo cardiometabólico y osteomuscular cuando las características de tipo, frecuencia, intensidad y duración permitan tiempos de recuperación adecuados y se encuentren acompañados de una nutrición y periodos de descanso óptimos; de lo contrario, el riesgo de aparición de enfermedades crónicas sería una limitante para recomendarlo.

La necesidad de establecer o estandarizar ciertos tipos de entrenamientos de acuerdo con las variables sociodemográficas de los individuos (tiempo de entrenamiento, grupos de edad, especificidad del curso, género, somatotipo, etc.) al igual que lograr una prescripción individualizada del entrenamiento, son aspectos que limitan la posibilidad de potencializar las habilidades de desempeño en las condiciones difíciles a las que se ve enfrentado el militar, ya sea en operaciones simuladas o reales.

Para finalizar, la pregunta por el entrenamiento físico militar óptimo requiere reflexiones permanentes acordes con las necesidades propias de las misiones, las cualidades y posibilidades de los sujetos y las instituciones, así como con los ámbitos específicos de acción y los desarrollos tecnológicos alcanzados.

\section{Referencias}

Arráez, M., Calles, J., \& Moreno, L. (2006). La hermenéutica: una actividad interpretativa. Sapiens. Revista universitaria de investigación, 7(2), 171-181. http://www.redalyc.org/articulo.oa?id=41070212

Crowley, S., Wilkinson, L., Burroughs, E., Muraca, S., Wigfall, L., Louis-Nance, T., Williams, E., Glover, S., \& Youngstedt, S. (2012). Sleep during basic combat training: a qualitative study. Military medicine, 177(7), 823-828.

Fedak, S. (2014). Physical examination performed by the international military operations in mountainous terrain. Pedagogics, psychology, medical-biological problems of physical training and sports, 5, 67-73. 
Friedl, K. (2015). U.S. Army research on pharmacological enhancement of soldier performance: stimulants, anabolic hormones, and blood doping. Journal of strength and conditioning research, 29, 71-76.

Gibala, M., Gagnon, P., \& Nindl, B. (2015). Military applicability of interval training for health and performance. Journal of strength and conditioning research, 29, 40-45.

Gist, N., Freese, E., Ryan, T., \& Cureton, K. (2015). Effects of low-volume, high-intensity whole-body calisthenics on army rotc cadets. Military medicine, 180(5), 492-498.

Gonçalves, M., Fonseca, M., Beltrame, J., \& Correa, J. (2016). Is it possible to identify underlying cardiovascular risk in young trained military? Journal of sports medicine and physical fitness, 56(1), 125-132.

Hofstetter, M., Mader, U., \& Wyss, T. (2012). Effects of a 7-week outdoor circuit training program on Swiss army recruits. Journal of strength and conditioning research, 26(12), 3418-3425.

Jameson, J., Pappa, L., McGuire, B., \& Kelly, K. (2015). Performance differences between male and female marines on standardized physical fitness tests and combat proxy tasks: identifying the gap. U.S. Army medical department journal, 12-21.

Kechijian, D., \& Rush, S. (2012). Tactical physical preparation: the case for a movement-based approach. Journal of special operations medicine: a peer reviewed journal for SOF medical professionals, 12(3), 43-49.

Kilen, A., Hjelvang, L., Dall, N., Kruse, N., \& Nordsborg, N. (2015). Adaptations to short, frequent sessions of endurance and strength training are similar to longer, less frequent exercise sessions when the total volume is the same. Journal of strength and conditioning research, 29, 46-51.

Kraemer, W., \& Szivak, T. (2012). Strength training for the warfighter. Journal of strength and conditioning research, 26(7), 107-118.

Larsson, H., Tegern, M., \& Harms-Ringdahl, K. (2012). Influence of the implementation of a comprehensive intervention programme on premature discharge outcomes from military training. Work, 42(2), 241-251. doi:10.3233/WOR-2012-1347

Lennemann, L., Sidrow, K., Johnson, E., Harrison, C., Vojta, C., \& Walker, T. (2013). The influence of agility training on physiological and cognitive performance. Journal of strength and conditioning research, 27(12), 3300-3309.

Lieberman, H., Karl, J., McClung, J., Williams, K., \& Cable, S. (2016). Improved mood state and absence of sex differences in response to the stress of army basic combat training. Applied psychology: health and well-being, 8(3), 351-363. doi:10.1111/aphw.12075

Lisman, P., O'Connor, F., Deuster, P., \& Knapik, J. (2013). Functional movement screen and aerobic fitness predict injuries in military training. Medicine and science in sports and exercise, 45(4), 636-643. https://doi.org/10.1249/MSS.0b013e31827a1c4c

Mardones, J. (1991). Filosofía de las ciencias humanas y sociales: materiales para una fundamentación cientifica (Vol. I). Anthropos Editorial.

Nogueira, E., Oporto, L., Nogueira, R., Martins, W., Fonseca, R., Lunardi, C., \& De Oliveira, R. (2016). Body composition is strongly associated with cardiorespiratory fitness in a large 
brazilian military firefighter cohort: the brazilian firefighters study. Journal of strength and sonditioning research, 30(1), 33-38. doi:10.1519/JSC.0000000000001039

O’Hara, R., Serres, J., Traver, K., Wright, B., Vojta, C., \& Eveland, E. (2012). The influence of nontraditional training modalities on physical performance: review of the literature. Aviation space and environmental medicine, 83(10), 985-990. https://doi. org/10.3357/ASEM.3376.2012

O’Neal, E., Hornsby, J., \& Kelleran, K. (2014). High-intensity tasks with external load in military applications: a review. Military medicine, 179(9), 950-954. https://doi.org/10.7205/ MILMED-D-14-00079

Pasiakos, S., Karl, J., Lutz, L., Murphy, N., Margolis, L., Rood, J., Cable, S., Williams, K., Young, A., \& McClung, J. (2012). Cardiometabolic risk in US Army recruits and the effects of basic combat training. PloS ONE, 7(2), 1-7. https://doi.org/10.1371/journal. pone.0031222

Podestá, M. (2016). La educación integral del líder militar moderno. La experiencia de la Academia Militar de West Point. Revista digital universitaria del Colegio Militar de la Nación, 1(3), 1-16. Recuperado de http://www.rediu.colegiomilitar.mil.ar/pdf/ReDiU_0103_art01-La educacion integral del lider militar moderno.pdf

Riiser, A., Ripe, S., \& Aadland, E. (2015). Five training sessions improves 3000 meter running performance. Journal of sports medicine and physical fitness, 55(12), 1452-1458.

Rojas, P. (2017). Doctrina Damasco: eje articulador de la segunda gran reforma del Ejército Nacional de Colombia. Revista cientifica General José Maria Córdova, 15(19), 105-129. https://doi.org/10.21830/19006586.78

Roos, L., Boesch, M., Sefidan, S., Frey, F., Mäder, U., Annen, H., \& Wyss, T. (2015). Adapted marching distances and physical training decrease recruits' injuries and attrition. Military medicine, 180(3), 329-336. doi:10.7205/MILMED-D-14-00184

Sell, T., Abt, J., Nagai, T., Deluzio, J., Lovalekar, M., Wirt, M., \& Lephart, S. (2016). The eagle tactical athlete program reduces musculoskeletal injuries in the 101st Airborne Division (Air Assault). Military medicine, 181(3), 250-257.

Sporiš, G., Harasin, D., Baić, M., Krističević, T., Krakan, I., Milanović, Z., Cular, D., \& Bagarić-Krakan, L. (2014). Effects of two different 5 weeks training programs on the physical fitness of military recruits. Collegium antropologicum, 38(2), 157-164.

Szivak, T., \& Kraemer, W. (2015). Physiological readiness and resilience: pillars of military preparedness. Journal of strength and conditioning research, 29(11), 34-39. https://doi. org/10.1519/JSC.0000000000001073

Theiss, J., Gerber, J., Cameron, K., Beutler, A., Marshall, S., Distefano, L., Padua, D., De la Motte, S., Miller, J., \& Yunker, C. (2014). Jump-landing differences between varsity, club, and intramural athletes: the Jump-ACL Study. Journal of strength and conditioning research, 28(4), 1164-1171. https://doi.org/10.1519/JSC.0b013e3182alfdcd

Tomczak, A., Bertrandt, J., Klos, A., \& Klos, K. (2016). Influence of military training and standardized nutrition in military unit on soldiers' nutritional status and physical fitness. Journal of strength and conditioning research, 30(10), 2774-2780. 
Vantarakis, A., Chatzinikolaou, A., Avloniti, A., Vezos, N., Douroudos, I., Draganidis, D., Jamurtas, A., Kambas, A., Kalligeros, S., \& Fatouros, I. (2017). A 2-month linear periodized resistance exercise training improved musculoskeletal fitness and specific conditioning of navy cadets. Journal of strength and conditioning research, 31(5), 1362-1370.

Vierling, K., Whittington, M., \& Cross, A. (2012). Challenges in developing HSCB standards and interoperability for marine corps training simulations. https://www.sisostds. org/DesktopModules/Bring2mind/DMX/API/Entries/Download?Command=Core_ Download \&EntryId $=35459 \&$ PortalId $=0 \&$ TabId $=105$

Vrijkotte, S., Meeusen, R., Roelands, B., Kubesch, S., Mairesse, O., De Schutter, G., \& Pattyn, N. (2017). Refining selection for elite troops by predicting military training outcome. Aerospace medicine and human performance, 88(9), 850-857. doi:10.3357/ AMHP.4818.2017

Wyss, T., Scheffler, J., \& Mäder, U. (2012). Ambulatory physical activity in swiss army recruits. International journal of sports medicine, 33(9), 716-722. https://doi. org/10.1055/s-0031-1295445 